\title{
Taxa de morbimortalidade entre homens e mulheres com diagnóstico de infarto agudo do miocárdio
}

\author{
Morbidity and mortality rate among men and women diagnosed with myocardial infarction
}

Tasa de morbimortalidad entre hombres y mujeres diagnosticados con infarto agudo del miocardio

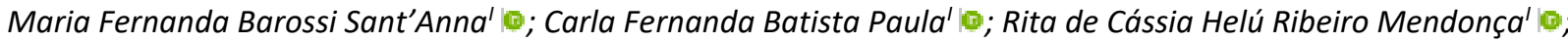

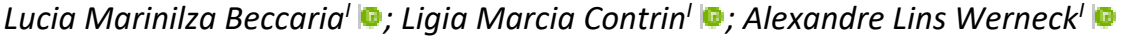 \\ 'Faculdade de Medicina de São José do Rio Preto, São José do Rio Preto, SP, Brasil
}

\begin{abstract}
RESUMO
Objetivo: verificar se existe diferença na taxa de morbimortalidade entre homens e mulheres com diagnóstico de infarto agudo do miocárdio em um hospital de ensino. Método: estudo transversal, descritivo e quantitativo, com amostra de 647 prontuários, comparando-se fatores de risco, dados sociodemográficos e referentes à internação hospitalar. Resultados: de 647 pacientes, 415 eram homens e 232 mulheres, com idade variando entre 61 e 80 anos. Dos fatores de risco, 74,96\% eram hipertensos em ambos os sexos, sendo que o percentual de hipertensão, diabetes e dislipidemia foi maior no sexo feminino. A taxa de mortalidade foi de $17,24 \%$ nas mulheres e $9,64 \%$ nos homens. Dentre os fatores de risco relacionados ao óbito, o mais significante foi a hipertensão arterial, seguido de diabetes e dislipidemia. Conclusão: houve maior prevalência de infarto do miocárdio no sexo masculino, entretanto, a taxa de morbidade e mortalidade foi maior no sexo feminino.

Descritores: Morbidade; Mortalidade; Homens; Mulheres; Infarto do Miocárdio.
\end{abstract}

\begin{abstract}
Objective: to identify any difference in morbidity and mortality rate between men and women diagnosed with acute myocardial infarction. Method: this quantitative, descriptive, cross-sectional study, involving a sample of 647 medical records, compared risk factors, and sociodemographic and hospitalization-related data. Results: of 647 patients, 415 were men and 232 women aged from 61 to 80 years. Risk factors included $74.96 \%$ of both sexes were hypertensives. Percentages of hypertension, diabetes, and dyslipidemia were higher in women. Mortality rates were $17.24 \%$ in women and $9.64 \%$ in men. The most significant risk factors for mortality were arterial hypertension, followed by diabetes and dyslipidemia. Conclusion: prevalence of myocardial infarction was higher in men, but morbidity and mortality rates were higher in women.
\end{abstract}

Descriptors: Morbidity; Mortality; Men; Women; Myocardial Infarction.

\section{RESUMEN}

Objetivo: : verificar si existe alguna diferencia en la tasa de morbimortalidad entre hombres y mujeres diagnosticados con infarto agudo de miocardio en un hospital universitario. Método: se trata de un estudio transversal, descriptivo y cuantitativo, que involucró una muestra de 647 historias clínicas, comparando factores de riesgo, datos sociodemográficos y relacionados con la hospitalización. Resultados: de 647 pacientes, 415 eran hombres y 232 mujeres con edades comprendidas entre 61 y 80 años. En cuanto a los factores de riesgo, el 74,96\% era hipertenso en ambos sexos. El porcentaje de hipertensión, diabetes y dislipidemia se mostró superior entre las mujeres. La tasa de mortalidad fue del 17,24\% en mujeres y del 9,64\% en hombres. Entre los factores de riesgo relacionados con la muerte, el más significativo fue la hipertensión arterial, seguido de la diabetes y la dislipidemia. Conclusión: hubo una mayor prevalencia de infarto de miocardio entre hombres, sin embargo, la tasa de morbilidad y mortalidad fue más alta entre mujeres.

Descriptores: Morbilidad; Mortalidad; Hombres; Mujeres; Infarto del Miocardio.

\section{INTRODUÇÃO}

O Infarto Agudo do Miocárdio (IAM), também chamado de ataque cardíaco, consiste na interrupção do fluxo sanguíneo por um determinado tempo, causando lesões ou morte das células do músculo cardíaco ${ }^{1,2}$. Ele faz parte das doenças cardiovasculares (DCV), responsáveis por aproximadamente $30 \%$ dos óbitos do mundo e considerada uma das principais causas de morte ${ }^{1,3-5}$. Destarte, as cardiopatias são consideradas um problema de saúde pública, além de gerarem alto custo referente às internações hospitalares no Brasil ${ }^{6,7}$.

Nos Estados Unidos, $53 \%$ das causas de morte são por doenças do coração, ocorrendo aproximadamente 782 mil casos de infarto por ano em americanos com mais de 65 anos $^{8}$. No Brasil, segundo o departamento de informática do Sistema Único de Saúde (DATASUS), em 2013, observou-se um aumento de 48\% de mortes por IAM entre 1996 e 2011 e, apenas em 2010, houve aproximadamente 79 mil óbitos relacionados a essa doença ${ }^{9,10}$. De acordo com o mesmo departamento, foram registrados 2.028 óbitos por IAM no estado de São Paulo, apenas no mês de agosto de $2013^{11}$.

Agradecimentos ao Programa Institucional de Bolsas de Iniciação Científica PIBIC-CNPq. Protocolo: 0001-002954/2018.

Autora correspondente: Maria Fernanda Barossi Sant'Anna. E-mail: mfernandabarossis@gmail.com

Editora Científica: Cristiane Helena Gallasch; Editor Associado: Antonio Marcos Tosoli Gomes 
Entre as doenças relacionadas a problemas do sistema cardiovascular estão a insuficiência cardíaca, acidente vascular cerebral (AVC), IAM, hipertensão arterial sistêmica (HAS) e arritmia como algumas das mais conhecidas. Os fatores de risco, quando não tratados e controlados, desencadeiam doenças no coração, portanto, de modo geral, além de fazer exames periódicos e seguir corretamente as orientações profissionais, é necessário adotar um estilo de vida saudável, que combine uma alimentação balanceada com a prática de exercícios físicos, controlar o peso, não fumar ou ingerir bebidas alcoólicas em excesso e evitar o estresse, melhorando a qualidade de vida e aumentando a sobrevida ${ }^{14}$.

Alguns fatores de risco relacionados ao IAM são intrínsecos, como sexo, genética, raça e idade ${ }^{12,13}$. Dor intensa no peito e aperto que irradia para os ombros, costas, mandíbula, pescoço e braços, sensação de desmaio, falta de ar, dor no estômago, tosse seca e constante, além de náusea, vômito e suor, geralmente depois de exercícios físicos, são alguns dos sinais e sintomas do IAM $^{14}$.

Das DCV, o IAM possui maior incidência e merece atenção especial, porque causam 6,5 vezes mais mortes do que todas as infecções (incluindo a ais) e mata dez vezes mais que o câncer de mama ${ }^{15,16}$. Persiste na sociedade o mito de que as DCV seriam apenas doenças que acometem os homens ${ }^{15}$

As complicações do IAM em mulheres podem ser ainda maiores do que nos homens, pela maior fragilidade das estruturas femininas. Quanto maior a extensão do infarto, mais frequentes e graves serão as complicações. Na mulher ocorre a queda na produção do estrogênio na menopausa, o que também facilita alterações na pressão arterial e no colesterol, fatores que levam ao IAM. O uso de pílulas anticoncepcionais, má alimentação, obesidade e até dupla jornada de trabalho, fazem com que o número de óbitos entre as mulheres aumente ${ }^{14}$.

$\mathrm{Na}$ faixa etária acima de 40 anos, as mulheres que sofrem um ataque cardíaco acumulam mais fatores de risco como diabetes, hipertensão e altas taxas de colesterol. Portanto, há necessidade de melhorar a conscientização sobre as doenças do coração, especialmente das mulheres, por meio de informações que visam estimular a adoção de hábitos saudáveis, evitando não só DCV, como também os fatores agravantes ${ }^{14}$.

Assim, torna-se oportuno uma pesquisa que identifique a prevalência de IAM e, se existe diferença entre homens e mulheres em relação à taxa de morbimortalidade, relacionando-se os fatores de risco, dados sociodemográficos e referentes à internação, para propiciar maior orientação e conscientização, principalmente de mulheres, sobre a importância da promoção e prevenção das DCV e do diagnóstico e tratamento precoce do IAM. Diante disso, o objetivo deste estudo foi verificar se existe diferença na taxa de morbimortalidade entre homens e mulheres com diagnóstico de IAM em um hospital de ensino.

\section{MÉTODO}

Estudo transversal, com delineamento descritivo e abordagem quantitativa, realizado por meio de prontuários eletrônicos de pacientes de ambos os sexos, acima de 18 anos, que estiveram internados no setor da cardiologia com diagnóstico de IAM, em um hospital de ensino do interior paulista, entre maio de 2016 a maio de 2018 (total de 24 meses).

Foram coletadas informações em 647 prontuários de pacientes, aqui tratados como amostra de uma população existente de 720 . Foram excluídos do estudo 73 prontuários incompletos ou que foram transferidos para outros setores e cidades.

Os dados foram analisados, comparando-se as características dessa população, de acordo com os fatores de risco, como tabagismo, dislipidemia (DLP), HAS, DM, história familiar e índice de massa corporal (IMC). Foram correlacionados dados sociodemográficos (sexo, estado civil, idade, cor, escolaridade, religião e profissão) e aqueles referentes à internação (necessidade de cirurgia, permanência em dias no hospital, tipo de convênio, origem da internação, alta ou óbito). O estudo foi aprovado pelo Comitê de Ética em Pesquisa da instituição de acordo com a Resolução no. 466/12.

O perfil da amostra foi caracterizado pela análise descritiva, contemplando as variáveis analisadas e seus desdobramentos. Os dados foram replicados de forma absoluta e relativa nesta primeira parte.

$\mathrm{Na}$ análise inferencial, delineou-se como objetivo estatístico, a análise de independência e predição entre as variáveis propostas no escopo do trabalho. Para isso, utilizou-se, dentro dos padrões esperados, os testes Regressão de Poisson e U de Mann-Whitney.

Os resultados de independência entre as variáveis propostas se deram pela análise do valor de P (significância). Todas as análises foram obtidas pelo Software SPSS Statistics (Versão 23) atreladas às funcionalidades da ferramenta Excel (versão 2.016).

$\mathrm{Na}$ análise da parte inferencial dos cruzamentos estatísticos, foram analisados os resultados do teste de normalidade das variáveis. Os métodos escolhidos para abordagem das análises de variação dos resultados entre os grupos analisados verificaram a relação entre eles, em que se parametrizou uma das variáveis como sendo dependente e a outra como independente, objetivando a análise de predição entre ambas. 
Para todas as análises, um valor de $\mathrm{P}<0,05$ foi considerado estatisticamente significante, caracterizando a significância entre os grupos estudados. Todos os testes contemplam erro alfa de $5 \%$ e confiabilidade de $95 \%$.

A normalidade foi testada com o teste de Kolmogorov-Smirnov. As diferenças entre as medianas foram avaliadas por meio dos testes não paramétricos, usando o modelo de regressão de Poisson que tem por característica a análise de dados contados na forma de proporções ou razões de contagem, ou seja, leva em consideração o total de pessoas com uma determinada doença.

Foram feitos testes de hipóteses, usando método Regressão de Poisson e U de Mann-Whitney, em que foram analisados o comportamento das correlações entre as variáveis analisadas e o grau de explicação da variável dependente em relação às variáveis independentes da amostra.

\section{RESULTADOS}

Considerou-se uma amostra de 647 prontuários eletrônicos de pacientes acima de 18 anos, internados no setor da cardiologia com diagnóstico de IAM, entre maio de 2016 a maio de 2018 em um hospital de ensino, da cidade de São José do Rio Preto, São Paulo. A amostra foi composta por 415 homens (64,14\%) e 232 mulheres (35,86\%) e, 52,40\% tinham idade entre 61 e 80 anos (média=64 anos), sendo que 57,76\% ( $n=134$ ) eram do sexo feminino e 49,40\% ( $n=205$ ) do sexo masculino. Além disso, $90,73 \%$ eram brancos, $57,50 \%$ casados, seguido de viúvos (17\%), 41,58\% tinham ensino fundamental (1 série). A maioria dos homens era ativo em suas profissões, enquanto a maioria das mulheres era inativa.

$\mathrm{Na}$ análise dos fatores de ricos versus sexo masculino e feminino, constatou-se que $74,96 \%$ dos pacientes eram hipertensos, com um percentual de $84,48 \%(n=196)$ de mulheres hipertensas e $69,64 \%(n=289)$ de homens hipertensos. Ainda, $60,43 \%$ eram diabéticos, sendo $52,16 \%$ do sexo feminino e $32,53 \%$ do sexo masculino e $28,44 \%$ ( $n=184$ ) eram tabagistas, 22,10\% ex-tabagistas, 20,87\% ( $n=135)$ possuíam dislipidemia, 6,34\% ( $n=41)$ tinham histórico na família de IAM, 37,09\% ( $n=240)$ estavam acima do peso e 19,78\% ( $n=128)$ eram obesos. O percentual de hipertensão, diabetes e dislipidemia foi maior no sexo feminino. O sexo masculino apresentou maior número de ex-tabagistas e tabagistas. $A$ tabela 1 mostra as variáveis sociodemográficas e fatores de risco significantes em relação a amostra.

TABELA 1: Análise dos dados sociodemográficos e dos fatores de risco versus sexo masculino e feminino. São José do Rio Preto, SP, Brasil. 2019

\begin{tabular}{|c|c|c|c|c|c|c|}
\hline \multirow[b]{2}{*}{ Variável } & \multicolumn{2}{|c|}{ Feminino } & \multicolumn{2}{|c|}{ Masculino } & \multicolumn{2}{|c|}{ Total } \\
\hline & $\mathbf{N}$ & $\%$ & $\mathbf{N}$ & $\%$ & $\mathbf{N}$ & $\%$ \\
\hline \multicolumn{7}{|l|}{ Estado Civil $p<0,000^{*}$} \\
\hline Casado (a) & 118 & 50,86 & 254 & 61,20 & 372 & 57,50 \\
\hline Desquitado (a) & 6 & 2,59 & 14 & 3,37 & 20 & 3,09 \\
\hline Divorciado (a) & 9 & 3,88 & 22 & 5,30 & 31 & 4,79 \\
\hline Não Identificado (a) & 0 & 0,00 & 2 & 0,48 & 2 & 0,31 \\
\hline Solteiro (a) & 12 & 5,17 & 38 & 9,16 & 50 & 7,73 \\
\hline União Estável & 14 & 6,03 & 48 & 11,57 & 62 & 9,58 \\
\hline Viúvo (a) & 73 & 31,47 & 37 & 8,92 & 110 & 17,00 \\
\hline \multicolumn{7}{|l|}{ Escolaridade $p=0,009 *$} \\
\hline Analfabeto & 49 & 21,12 & 44 & 10,60 & 93 & 14,37 \\
\hline Somente Alfabetizado & 1 & 0,43 & 2 & 0,48 & 3 & 0,46 \\
\hline Fundamental- 1a a 4a & 89 & 38,36 & 180 & 43,37 & 269 & 41,58 \\
\hline Fundamental-5a a $8^{a}$ & 46 & 19,83 & 88 & 21,20 & 134 & 20,71 \\
\hline Ensino Médio & 37 & 15,95 & 65 & 15,66 & 102 & 15,77 \\
\hline Ensino Superior & 10 & 4,31 & 34 & 8,19 & 44 & 6,80 \\
\hline Especialização & 0 & 0,00 & 2 & 0,48 & 2 & 0,31 \\
\hline \multicolumn{7}{|l|}{ Profissão $p<0,000 * *$} \\
\hline Inativo & 177 & 76,29 & 50 & 12,05 & 227 & 35,09 \\
\hline Ativo & 55 & 23,71 & 365 & 87,95 & 420 & 64,91 \\
\hline \multicolumn{7}{|l|}{ Diabetes $p<0,000^{* *}$} \\
\hline Sim & 121 & 52,16 & 135 & 32,53 & 256 & 60,43 \\
\hline Não & 111 & 47,84 & 280 & 67,47 & 391 & 39,57 \\
\hline \multicolumn{7}{|l|}{ Ex-Tabagista $p=0,012 * *$} \\
\hline Sim & 35 & 15,09 & 108 & 26,02 & 143 & 22,10 \\
\hline Não & 197 & 84,91 & 307 & 73,98 & 504 & 77,90 \\
\hline TOTAL & 232 & 100 & 415 & 100 & 647 & 100 \\
\hline
\end{tabular}

*Teste de Mann-Whitney; **Análise de Regressão de Poisson 
Em relação ao tempo de internação e diabetes, 18 pacientes ficaram internados por mais de 30 dias; destes, 10 eram mulheres e 8 homens. Analisando-se os dados sociodemográficos e fatores de risco versus óbitos, percebeu-se que o número de óbitos foi pequeno entre os gêneros, com o percentual de $17,24 \%(n=40)$ do sexo feminino e $9,64 \%$ $(n=40)$ do masculino. Dos pacientes que morreram, $72,50 \%(n=58)$ tinham entre 61 a 80 anos, 41,25\% possuíam ensino fundamental da 1 a a 4 a série, $25 \%$ eram analfabetos, $56,25 \%$ estavam inativos, $52,50 \%$ ( $n=42$ ) estavam acima do peso e $10 \%(n=8)$ eram obesos. Dentre os óbitos, 59 (73,75\%) eram hipertensos, ou seja, foi a principal causa de mortalidade, sendo que 37 (46,25\%) dos óbitos, o diabetes foi a principal comorbidade, como demonstra a tabela 2.

TABELA 2: Análise dos dados sociodemográficos e fatores de risco versus altas e óbitos. São José do Rio Preto, SP, Brasil. 2019.

\begin{tabular}{|c|c|c|c|c|c|c|}
\hline \multirow[t]{2}{*}{ Variável } & \multicolumn{2}{|c|}{ Alta } & \multicolumn{2}{|c|}{ Óbito } & \multicolumn{2}{|c|}{ Total } \\
\hline & $\mathbf{N}$ & $\%$ & $\mathbf{N}$ & $\%$ & $\mathbf{N}$ & $\%$ \\
\hline \multicolumn{7}{|l|}{ Sexo $p=0,945^{*}$} \\
\hline Feminino & 192 & 82,76 & 40 & 17,24 & 232 & 35,86 \\
\hline Masculino & 375 & 90,36 & 40 & 9,64 & 415 & 64,14 \\
\hline \multicolumn{7}{|l|}{ Escolaridade $\mathrm{p}=0,001^{* *}$} \\
\hline Analfabeto & 73 & 12,87 & 20 & 25,00 & 93 & 14,37 \\
\hline Somente Alfabetizado & 2 & 0,35 & 1 & 1,25 & 3 & 0,46 \\
\hline Fundamental 1ㅁa 4 aa & 236 & 41,62 & 33 & 41,25 & 269 & 41,58 \\
\hline Fundamental 5aa 8aㅗ & 118 & 20,81 & 16 & 20,00 & 134 & 20,71 \\
\hline Ensino Médio & 93 & 16,40 & 9 & 11,25 & 102 & 15,77 \\
\hline Ensino Superior & 43 & 7,58 & 1 & 1,25 & 44 & 6,80 \\
\hline Especialização & 2 & 0,35 & 0 & 0,00 & 2 & 0,31 \\
\hline \multicolumn{7}{|l|}{ Profissão $p=0,022 *$} \\
\hline Inativo & 182 & 32,10 & 45 & 56,25 & 227 & 35,09 \\
\hline Ativo & 385 & 67,90 & 35 & 43,75 & 420 & 64,91 \\
\hline \multicolumn{7}{|l|}{ Hipertensão $p=0,016^{*}$} \\
\hline Sim & 426 & 75,13 & 59 & 73,75 & 485 & 74,96 \\
\hline Não & 141 & 24,87 & 21 & 26,25 & 162 & 25,04 \\
\hline TOTAL & 567 & 100 & 80 & 100 & 647 & . 100 \\
\hline
\end{tabular}

*Análise de Regressão de Poisson; **Teste de Mann-Whitney

A análise dos dados sobre a internação mostrou que em $66,92 \%$ ( $n=433)$ dos pacientes, o tempo de internação foi entre 1 a 10 dias, 81,45\% ( $n=527$ ) foram atendidos pelo sistema único de saúde (SUS), 72,02\% ( $n=466$ ) tiveram como porta de entrada o pronto atendimento da emergência e $73,11 \%(n=473)$ foram submetidos à procedimentos invasivos como revascularização do miocárdio ou angioplastia coronariana.

Referente à análise dos dados sobre a internação versus óbitos, o tempo de internação de 1 a dez dias teve maior número de óbitos ( $n=31 / 38,75 \%)$, seguido do tempo de internação menor que 1 dia-24 horas ( $n=23 / 28,75 \%)$. A maioria dos óbitos foi em pacientes do SUS (93,75\%), 82,50\% encaminhados pelo pronto atendimento da emergência. A Tabela 3 mostra que 50\% dos pacientes que morreram, precisaram de alguma cirurgia/procedimento (revascularização do miocárdio ou angioplastia coronariana).

TABELA 3: Análise dos dados sobre a internação versus altas e óbitos. São José do Rio Preto (SP), Brasil. 2019.

\begin{tabular}{|c|c|c|c|c|c|c|}
\hline \multirow[t]{2}{*}{ VARIÁVEL } & \multicolumn{2}{|c|}{ Alta } & \multicolumn{2}{|c|}{ Óbito } & \multicolumn{2}{|c|}{ Total } \\
\hline & $\mathbf{N}$ & $\%$ & $\mathbf{N}$ & $\%$ & $\mathbf{N}$ & $\%$ \\
\hline \multicolumn{7}{|l|}{ SUS ou Convenio $p=0,007^{*}$} \\
\hline SUS & 452 & 79,72 & 75 & 93,75 & 527 & 81,45 \\
\hline Convenio & 115 & 20,28 & 5 & 6,25 & 120 & 18,55 \\
\hline \multicolumn{7}{|c|}{ Origem do atendimento $p=0,011^{* *}$} \\
\hline Ambulatório SUS & 2 & 0,35 & 0 & 0,00 & 2 & 0,31 \\
\hline Emergência SUS & 29 & 5,11 & 4 & 5,00 & 33 & 5,10 \\
\hline Pronto atendimento SUS & 400 & 70,55 & 66 & 82,50 & 466 & 72,02 \\
\hline Internação SUS & 22 & 3,88 & 5 & 6,25 & 27 & 4,17 \\
\hline Emergência Convenio & 90 & 15,87 & 3 & 3,75 & 93 & 14,37 \\
\hline Internação Convenio & 24 & 4,23 & 2 & 2,50 & 26 & 4,02 \\
\hline \multicolumn{7}{|l|}{ Cirurgias $p<0,000^{*}$} \\
\hline Não & 134 & 23,63 & 40 & 50,00 & 174 & 26,89 \\
\hline Sim & 433 & 76,37 & 40 & 50,00 & 473 & 73,11 \\
\hline TOTAL & 567 & 100 & 80 & 100 & 647 & 100 \\
\hline
\end{tabular}


Entre os 80 óbitos registrados, 33 tinham ensino fundamental da 1a a 4a série e 15 eram mulheres e analfabetas, assim como os 5 óbitos em homens. Além disso, 36 mulheres que morreram estavam inativas quanto as suas profissões, enquanto 31 homens que morreram, estavam ativos. Entre os pacientes que morreram, tendo como a principal comorbidade o diabetes, 22 eram mulheres e 15 eram homens. Em relação à hipertensão, morreram 35 mulheres e 24 homens.

\section{DISCUSSÃO}

Entre os 647 pacientes, a maioria foi do sexo masculino e idade de 61 a 80 anos. Um estudo que comparou as regiões do Brasil, mostrou que na região Sudeste, a média de idade entre pacientes hospitalizados com IAM foi de 63,1 anos, sendo $66,4 \%$ do sexo masculino ${ }^{17}$. Na região Nordeste, observou-se também a predominância do sexo masculino, com um pequeno aumento na idade, o que pode estar diretamente relacionado a fatores genéticos e culturais ${ }^{18}$. Outro estudo mostrou que a faixa etária de $60 \pm 10$ anos, apresentou maior ocorrência de IAM ${ }^{19}$. O que corrobora com este estudo, visto que a prevalência de IAM foi maior em homens e faixa etária acima de 61 anos.

Quanto aos fatores de risco, 74,96\% eram hipertensos, 60,43\% diabéticos; $20,87 \%$ dislipidêmicos e a prevalência de tabagismo e ex-tabagista foi maior no sexo masculino. Outros estudos mostraram que, na região Sudeste, 70,9\% dos pacientes apresentavam quadro de HAS e $34,6 \%$ de dislipidemia (DLP) e, em 55,4\% dos casos, havia tabagistas atuais ou prévios ${ }^{17}$. Um estudo mostrou que durante a admissão hospitalar, 2/3 dos pacientes eram hipertensos e diabéticos ${ }^{20}$. Sedentarismo, obesidade e histórico familiar são outros fatores de risco prevalente na população idosa ${ }^{3}$.

Neste estudo, houve prevalência da HAS e DM nos pacientes com diagnóstico de IAM, ou seja, são fatores de risco que precisam de controle, acompanhamento médico e da adesão ao tratamento pela população. $O$ fator de risco diabetes teve significância com $p<0,000$ e segundo a V Diretriz da Sociedade Brasileira de Cardiologia sobre tratamento do IAM com supradesnível do segmento ST, ela é uma doença de desordem metabólica complexa que propicia o desenvolvimento da aterosclerose, sendo assim, os pacientes com diabetes evoluem com maior facilidade para o desenvolvimento de cardiopatia isquêmica e consequentemente, para uma maior morbimortalidade ${ }^{14}$.

Em uma revisão de literatura, os fatores de risco mais prevalentes para o IAM foram HAS, DM e tabagismo ${ }^{21}$. A HAS foi fator de maior prevalência na maioria dos estudos dessa revisão, exceto um realizado no Chile, em que a prevalência do fator tabagismo foi maior, com taxas variando entre $20 \%$ em países como Brasil e EUA, chegando à índices alarmantes de $36,5 \%$ no Chile. É importante destacar que o tabagismo é influenciado por fatores culturais e pode variar o consumo conforme a região ${ }^{22}$. Visto que o fator de risco mais prevalente neste estudo foi a HAS, seguido de DM e posteriormente tabagismo com $28,44 \%$. Segundo as diretrizes da sociedade de cardiologia, a prevenção secundária ao tabagismo recomenda o aconselhamento médico e caso seja necessário, o encaminhamento ao programa de cessação do tabagismo e a utilização de agentes farmacológicos, incluindo adesivos de nicotina ${ }^{14}$.

Neste estudo, verificou-se que a prevalência de infarto foi maior em homens, entretanto, a taxa de mortalidade foi maior em mulheres $(17,24 \%)$. Uma das possíveis explicações, se dá pelo fato de as mulheres terem características cardiovasculares diferentes das dos homens, como artérias e veias mais finas, o que faz com que a obstrução por placas ateroscleróticas seja mais grave. Outra razão é a presença do hormônio estrógeno, que é fator protetor para as mulheres, pois sua função vasodilatadora evita o acumula de LDL. Mas, na menopausa, ocorre a diminuição desse hormônio, tornando assim um episódio cardíaco pior nas mulheres ${ }^{20,23}$.

Os resultados dos estudos citados acima corroboram com o aumento da taxa de morbimortalidade em mulheres evidenciado neste estudo, principalmente após a menopausa e com o avanço da idade (fator de risco não modificável para doenças cardiovasculares). A diretriz da sociedade de cardiologia reforça os benefícios do tratamento com estatina na dislipidemia, como por exemplo a redução no número de mortes por doença arterial coronariana (DAC) ${ }^{14}$.

Sintomas como dores nas costas, náusea e queimação no estômago nem sempre são relacionados ao coração pelas mulheres. Geralmente, elas associam a problemas ortopédicos e gastrointestinais e, consequentemente, acabam retardando a procura por socorro médico ${ }^{23}$. Algumas vezes, dor na boca do estômago, nas costas e que sobe para a nuca, são também alguns dos sintomas de IAM que merecem atenção ${ }^{14}$.Neste sentido, é de suma importância a educação em saúde e conhecimento da população sobre os sintomas do IAM, para que assim, busquem imediatamente o serviço de saúde mais próximo.

A prevalência de pacientes hipertensos e diabéticos foi maior no sexo feminino, com idade entre 61 e 80 anos, em ambos os sexos. Um estudo mostra que a HA e o DM foram os fatores de risco mais prevalentes em mulheres do que nos homens, o que corrobora com este estudo. Além das mulheres procurarem os serviços de saúde mais tardiamente, exibem uma idade mais avançada do que a dos homens em 6,3 anos e, com isso, acabam apresentando mais comorbidades e alta letalidade ${ }^{24}$.

$\mathrm{O}$ atendimento rápido impacta na taxa de mortalidade. Em meio a pandemia do COVID-19, muitas pessoas têm demorado a agir quando passa mal, o que tem provocado o aumento em mortes em casa em todo o país. Na cidade de 
São José do Rio Preto - SP, os óbitos em casa por causa cardiovascular, aumentaram 64\% durante a quarentena, em relação a 2019. Houve uma redução de $57 \%$ dos óbitos por IAM constatadas nas emergências hospitalares segundo os dados da Associação dos Registradores de Pessoas Naturais do Estado de São Paulo (Arpen- SP) ${ }^{14}$.

Em relação aos fatores de risco versus sexo masculino e feminino, 37,09\% estavam acima do peso e 19,78\% eram obesos. Um estudo mostrou que a obesidade é também um fator de risco para o desenvolvimento de doenças crônicas ${ }^{25}$. O diagnóstico de sobrepeso corresponde a um IMC (Índice de Massa Corporal) $\geq 25 \mathrm{~kg} / \mathrm{m} 2$ e $<30 \mathrm{~kg} / \mathrm{m} 2$ e o de obesidade, IMC $\geq 30 \mathrm{~kg} / \mathrm{m} 2$, fatores de risco modificáveis que podem ser acompanhados na atenção primária, por meio de adesão ao tratamento, além de incentivar a alimentação equilibrada e atividade física ${ }^{14}$

Quanto aos dados sobre a internação versus óbitos, 28,75\% dos pacientes morreram em menos de $24 \mathrm{~h}$. Mostrouse em um estudo que a infraestrutura hospitalar é um fator preditor de desfecho do IAM, ou seja, o tempo para a admissão hospitalar é em média de 4 horas, o que acaba influenciando no desfecho clínico do paciente ${ }^{26}$. Dessa forma, o reconhecimento precoce dos sintomas, a busca imediata pelo serviço de emergência e a admissão são fatores que impactam na taxa de mortalidade como identificado neste estudo.

Analisando os fatores de risco versus óbitos, identificou-se que 73,75\% eram pacientes portadores de HA. Em um estudo realizado no setor de Urgência e Emergência, na Bahia, mostrou que a HAS foi um fator de risco mais prevalente e que evidenciou um índice expressivo de mortalidade ${ }^{5}$. Segundo as diretrizes da sociedade de cardiologia, a HAS contribui para a remodelação ventricular, aceleração da aterosclerose e a insuficiência cardíaca, dessa forma, a meta de controle estabelecida é de cifras $<140 / 90 \mathrm{mmHg}$, juntamente com a adesão ao tratamento medicamentoso ${ }^{14}$.

A taxa de óbito feminino e masculino foi, respectivamente, 17,24 e 9,64\%. Confirmou-se em outro estudo, que as taxas de morbimortalidade por IAM no Brasil ainda são elevadas. Um dos prováveis fatores é a dificuldade de acesso aos serviços especializados após os primeiros sintomas e pela falta de adesão e retardo ao tratamento no nível de atenção primaria ${ }^{27}$. Além da educação em saúde por parte dos profissionais, é necessário o entendimento e conscientização da população, visto que dentre os óbitos a escolaridade predominante foi ensino fundamental incompleto (41,25\%), seguido de analfabetos (25\%).

Um estudo mostrou que, entre 2008 a 2016, houve 21.398 casos de óbitos por IAM ocorridos no Brasil entre homens e mulheres de 30 a 59 anos. Na maioria das regiões, o sexo masculino apresentou maior quantidade de mortes notificadas com 13.587 óbitos, o que pode estar relacionada a essa faixa etária menor, visto que 57,76\% das mulheres deste estudo possuíam idade entre 60 e 80 anos $^{16}$.

Neste estudo, pode-se observar a importância da educação em saúde, prevenção e promoção na atenção primária, visto que vários fatores de risco são significativos para o IAM e que apesar dos homens internarem mais, as mulheres apresentaram taxa de mortalidade maior, mais comorbidades (HAS e DM) e uma idade mais avançada. Além disso, a aderência aos programas de reabilitação cardíaca após IAM é importante para os cardiopatas retornarem à vida produtiva, prevenir a progressão ou reverter o processo aterosclerótico, controlar os fatores de risco (hipertensão, diabetes, dislipidemia, obesidade, entre outros), reduzir morbimortalidade cardiovascular e melhorar os sintomas de angina, sendo que a prescrição da atividade física é de acordo com o acometimento e as características físicas de cada individuo ${ }^{14}$.

Pela utilização de prontuários eletrônicos, a falta de alguns dados relativos ao paciente como fatores estressores ou nível de ansiedade foi um fator limitante para o estudo.

\section{CONCLUSÃO}

A taxa de morbimortalidade de pacientes com IAM foi diferente entre homens e mulheres, visto que a prevalência foi maior no sexo masculino e a taxa de mortalidade foi maior no feminino. Ou seja, constatou-se que os homens internaram mais do que as mulheres durante o período da coleta de dados, porém, morreram mais mulheres.

Quanto ao perfil dos pacientes com IAM, a faixa etária com maior mortalidade foi entre 60 e 80 anos, com tempo de internação menor que 10 dias e a HAS foi o fator de risco mais significante em relação ao óbito, com maior prevalência no sexo feminino, seguido do DM. Verificou-se maior percentual de tabagismo e ex-tabagismo no sexo masculino.

O conhecimento do perfil de homens e mulheres que sofreram IAM propicia o desenvolvimento e fortalecimento das estratégias de prevenção tanto em nível hospitalar quanto ambulatorial. Alguns exemplos são: capacitação da equipe de saúde sobre as evidências científicas em relação as DCV e o perfil da população acometida para propor ações para uma assistência mais efetiva; ações educativas de promoção e prevenção à saúde sobre a importância da adesão ao tratamento medicamentoso e do controle dos fatores de risco como a HAS, DM, tabagismo, obesidade, estresse e o estímulo a hábitos de vida mais saudáveis, como alimentação equilibrada e a prática de atividade física, além do acompanhamento de consultas e exames de rotina e capacitar a equipe e a população sobre o atendimento de urgência 
no molde de suporte básico de vida (BLS). No entanto, há necessidade de novos estudos relacionando fatores de risco, variáveis sociodemográficas e referentes à internação hospitalar em homens e mulheres com IAM.

\section{REFERÊNCIAS}

1. Silveira DS, Jaeger CP, Hatschbach L, Manenti ERF. Validation of TIMI Risk Score for STEMI. Int. J. Cardiovasc. Sci. [Internet]. 2016 [cited 2020 Jul 21]; 29(3):189-97. DOI: https://doi.org/10.5935/2359-4802.20160034.

2. Vargas RA, Riegel F, Oliveira Junior N, Siqueira DS, Crossetti MGO. Quality of life of patients after myocardial infarction: literature integrative review. Rev Enferm UFPE on line [Internet]. 2017 [cited 2020 Jul 21]; 11(7):2803-9. Available from: https://periodicos.ufpe.br/revistas/revistaenfermagem/article/view/23456.

3. World Health Organization [Internet]. Geneve: WHO; 2016 [cited 2019 Jun 26]. Cardiovascular diseases. Available from: https://www.who.int/cardiovascular_diseases/en/.

4. Mansur AP, Favarato D. Trends in Mortality Rate from Cardiovascular Disease in Brazil, 1980-2012. Arq. Bras. Cardiol. [Internet]. 2016 [cited 2020 Jul 21]; 107(1):20-5. DOI: https://doi.org/10.5935/abc.20160077.

5. Araújo IFM, Santos ISC, Longuiniere ACFL, Valença-Neto PF, Franklin TA. Profile of the population affected by acute myocardial infarction. Rev. Enferm. UFPE on line [Internet]. 2016 [cited 2020 Jul 21]; 10(7):2302-9. Available from: https://periodicos.ufpe.br/revistas/revistaenfermagem/article/view/11284.

6. Siqueira ASE, Siqueira-Filho AG, Land MGP. Analysis of the Economic Impact of Cardiovascular Diseases in the Last Five Years in Brazil. Arq. Bras. Cardiol. [Internet]. 2017 [cited 2020 Jul 21]; 109(1):39-46. DOI: https://doi.org/10.5935/abc.20170068.

7. Ribeiro AL, Duncan BB, Brant LC, Lotufo PA, Mill JG, Barreto SM. Cardiovascular health in Brazil: Trends and perspectives. Circulation [Internet]. 2016 [cited 2020 Jul 21]; 133(4):422-33. DOI: https://doi.org/10.1161/CIRCULATIONAHA.114.008727.

8. Hom T, Haase N, Rosamend W, Howard VJ, Rumsfeld J, ManolioT, et al. Heart Disease and Stroke Statistics-2006 Update update: a report from the American Heart Association Statistics Committee and Stroke Statistics Subcommittee. Circulation [Internet]. 2006 [cited 202021 Jul]; 113(6):e85-151. DOI: https://doi.org/10.1161/CIRCULATIONAHA.105.171600.

9. Schmidt MM, Quadros AS, Martinelli ES, Gottschall CAM. Prevalence, etiology, and characteristics of patients with type-2 acute myocardial infarction. Rev. Bras. Cardiol. Invasiva [Internet]. 2015 [cited 2020 Jul 21]; 23(2):119-23. DOI: https://doi.org/10.1016/j.rbci.2015.12.010.

10. RIPISA. Ministério da Saúde [Internet]. 2000 [cited 2017 Jul 23]. Indicadores de Mortalidade: Taxa de Mortalidade por Doenças do aparelho Circulatório Ficha de qualificação. Available from: http://tabnet.datasus.gov.br/cgi/idb2000/fqc11.htm.

11. DATASUS [Internet]. São Paulo: DATASUS; C2019. Dados do DATASUS demonstram o número de casos de Infarto Agudo do Miocárdio em São Paulo [about 2 screens]. [cited 2019 Jun 20]. Available from: http://datasus.saude.gov.br/nucleosregionais/sao-paulo/noticias-sao-paulo/602-dados-do-datasus-demonstram-o-numero-de-casos-de-infarto-agudo-domiocardio-em-sao-paulo.

12. Carvalho CA, Fonseca PCA, Barbosa JB, Machado SP, Santos AM, Silva AAM. The association between cardiovascular risk factors and anthropometric obesity indicators in university students in São Luís in the State of Maranhão, Brazil. Ciênc. Saúde Coletiva [Internet]. 2015 [cited 2020 Jul 21]; 20(2):479-90. DOI: https://doi.org/10.1590/1413-81232015202.02342014.

13. Paz MP, Peres MB. Prevalência do Infarto Agudo do Miocárdio (IAM) no Município de Xanxere - SC: Benefícios da Reabilitação Cardiovascular na Fase 1 - Hospitalar [Internet]. 2016. [cited $2020 \mathrm{Jul} 21$ ]; Available from: http://www.uniedu.sed.sc.gov.br/wp-content/uploads/2016/09/Milene-Paz-e-Paz.pdf.

14. V Diretriz da Sociedade Brasileira de Cardiologia sobre Tratamento do Infarto Agudo do Miocárdio com Supradesnível do Segmento ST. Arq. Bras. Cardiol. [Internet]. 2015. [cited 2020 Jul 21]; Available from: http://publicacoes.cardiol.br/2014/diretrizes/2015/02_TRATAMENTO\%20DO\%20IAM\%20COM\%20SUPRADESNIVEL\%20DO\%20 SEGMENTO\%20ST.pdf.

15. Bortoleto G. Mulheres, está na hora de cuidar do coração. Diário da Região. 24 set 2017; Seção Bem Estar:4.

16. Medeiros TLF, Andrade PCNS, Davim RMB, Santos NMG. Mortality by an acute myocardial infarction. Rev. Enferm. UFPE on line [Internet]. 2018 [cited 2020 Jul 21]; 12(2):565-72. DOI: https://doi.org/10.5205/1981-8963-v12i2a230729p565-572-2018.

17. Nicolau JC, Franken M, Lotufo PA, Carvalho AC, Marin Neto JA, Lima, FG, et al. Use of demonstrably effective therapies in the treatment of acute coronary syndromes: comparison between different brazilian regions. Analysis of the Brazilian Registry on Acute Coronary Syndromes (BRACE). Arq. Bras. Cardiol. [Internet]. 2014 [cited 2020 Jul 21]; 98(4):282-9. Available from: https://www.scielo.br/pdf/abc/v98n4/en_v98n4a01.pdf.

18. Rosa RS, Macêdo DA, Oliveira BG, Bomfim ES, Casotti CA, Prado IF. Evidências para o cuidado de enfermagem na avaliação do risco coronariano em pacientes hospitalizados. Rev. Pesqui. Cuid. Fundam. (Online) [Internet]. 2016 [cited 2020 Jul 21]; 8(2):4460-71. DOI: https://doi.org/10.9789/2175-5361.2016.v8i2.4460-4471.

19. Marques MCMP, Mendes FRP, Serra ICC. Lifestyles: social representations construed by patients with myocardial infarction and family members. Rev. Gaúcha Enferm. [Internet]. 2017 [cited 2020 Jul 21]; 38(2): e62593. DOI: http://dx.doi.org/10.1590/19831447.2017.02.62593.

20. Franken M. Avaliação das variáveis de desempenho no tratamento das síndromes isquêmicas miocárdicas estáveis no Brasil: análise do registro BRACE (Brazilian Registry in Acute Coronary Syndromes) [tese de doutorado]. São Paulo: Faculdade de Medicina - Universidade de São Paulo; 2016.

21. Pinheiro RHO, Lenhani BE, Martins EV. Prevalence of risk factors related to acute myocardial infarction in elderly patients: an integrative review. Rev. UNINGÁ [Internet]. 2017 [cited 2018 Nov 28]; 30(3):83-8. Available from: http://revista.uninga.br/index.php/uningareviews/article/view/2023/1616. 
22. Daniel M, Ekenbäck C, Agewall S, Brolin EB, Caidahl K, Cederlund K, et al. Risk factors and markers for acute myocardial infartion with angiographically normal coronary arteries. Am. J. Cardiol. [Internet]. 2015 [cited 2020 Jul 21]; 116(6):838-44. DOI: https://doi.org/10.1016/j.amjcard.2015.06.011.

23. HCOR. Mulheres tem $50 \%$ de probabilidade de infarto quanto comparada aos homens. HCOR, Associação Brasileira Síria [Internet]. São Paulo: HCOR; 2017 [cited 2019 Jun 20]. Available from: https://www.hcor.com.br/imprensa/noticias/mulherestem-50-de-probabilidade-de-infarto-maior-quando-comparada-aos-homens/.

24. Mertins SM, Kolankiewicz ACB, Rosanelli CLSP, Loro MM, Poli G, Winkelmann ER, et al. Prevalence of risk factors in patients with acute myocardial infarction. Av. Enferm. [Internet]. 2016 [cited $2020 \mathrm{Jul} 21$ ]; 34(1):30-38. Available from: https://revistas.unal.edu.co/index.php/avenferm/article/view/37125/56822.

25. Katz M, Bosworth HB, Lopes RD, Dupre ME, Morita F, Pereira C, et al. A time-series analysis of the relation between unemployment rate and hospital admission for acute myocardial infarction and stroke in Brazil over more than a decade. Int J Cardiol [Internet]. 2016 [cited 2020 Jul 21]; 224:33-6. DOI: https://doi.org/10.1016/j.ijcard.2016.08.309.

26. Tateiva RARF. Epidemiological profile of deaths from and myocardial infarction in men by region of Santa Catarina [Internet]. 2016 [cited 2019 Jun 28]. Available from: http://www.uniedu.sed.sc.gov.br/wp-content/uploads/2016/09/RobertaFruscalso.pdf.

27. Santos J, Meira KC, Camacho AR, Salvador PTCO, Guimarães RM, Pierin AMG, et al. Mortality due to acute myocardial infarction in Brazil and its geographical regions: analyzing the effect of age-period-cohort. Ciênc. Saúde Coletiva [Internet]. 2018 [cited 2020 Jul 21]; 23(5):1621-34. DOI: https://doi.org/10.1590/1413-81232018235.16092016. 